\title{
An Improved Sequential Smoothing Particle Filtering Method
}

\author{
Shijie Cao* \\ University of Electronic Science and Technology of China, 2006 Xiyuan Ave, West Hi-Tech Zone
}

\begin{abstract}
In order to cope with the challenges of non-cooperative targets such as stealth targets to modern radar, especially when traditional threshold detection and tracking methods can hardly detect fast-moving stealth targets, technological innovation has long been required. In this paper we have proposed a new algorithm which can reduce computational cost and improve tracking accuracy. Firstly, the number of particles in the traditional particle filter is reduced and a small number of sampling points are derived from the possible distribution of the target to be tracked, each given a proper weight. Then, the transformed sampling points are sequentially smoothed. And finally, the target positions are estimated. The simulation results show that the proposed algorithm is more accurate than the traditional particle filter algorithm and has lower computational complexity. In the case when SNR is between 0dB to $15 \mathrm{~dB}$, a total of 100 Monte Carlo simulations are carried out, obtaining a high detection probability. The detection probability of the improved algorithm is higher than that of the existing particle filter at $7 \mathrm{~dB}$. Also, the computational cost is lower than the existing particle filter algorithm. Keywords: Smoothing, Particle Filter, Tracking
\end{abstract}

\section{Introduction}

Military combat technologies, equipment and combat methods have been improving with the development of social progress and continuous innovation. Having experienced several local wars and regional conflicts since the 90s, many countries and regions in the world are developing and improving military equipment to adapt to the new and complicated environment of modern war. Among them, the radar is an important "radio detection and ranging" instrument, making use of the reflected waves from the target echo to extract information including distance, angle, speed and so on [1]. In a word, in modern warfare, the strategic radar technology is significant.

In recent decades, due to the application of digital electronic computers and the emergence of new theory, new technologies and devices, the development of radar technology has undergone a fundamental change [2]. Along with these changes, the main threats to modern radar come from many aspects such as radiation-absorbent materials and stealth technology, anti-radiation missiles, integrated electronic interference, rapid ultra-low altitude penetration and so on. In order to cope with the challenges of non-cooperative targets such as stealth targets to modern radar, passive location and tracking methods are booming [3]. And a key problem in passive system signal detection technology is the detection and tracking of weak maneuvering targets $[4,5]$.

The term weak especially refers to the low reflection cross-sectional area of the radar, generates from low detectable targets with weak electromagnetic reflections. These targets include stealth aircrafts, missiles, small ships, and so on [6]. When these targets are irradiated with electromagnetic waves, the relative intensity of the reflected waves are significantly reduced compared with traditional radar targets. The resulting echo signals are very weak and the signal to noise ratios are also very low, which can reduce the detection range of an ordinary single base radar by one third or even half $[7,8]$. At the same time, these typical weak targets tend to have very high mobility. Target maneuvering refers to the phenomenon when the change of the original movement of the target go beyond what the filter model can track, which can usually cause model mismatch. The combination of the weak target echo and the maneuverability of the motion not only shortens the detection range of the traditional radar, but also greatly reduces the sensitivity of the detection [9]. Therefore, the traditional threshold detection and tracking method for the weak maneuvering target is not at an advantage. As a result, technological innovations have been imminent at hand

\section{Problem Formulation}

Several hypotheses about the target must be satisfied before it can be tracked. The target is in uniform media space, the state and density of which is time invariant. In addition, the target state can be described by a finite state space hidden Markov model. Moreover, the model's parameters are known. Based on the above assumptions, the detection and tracking process can be summarized and illustrated as figure 1 .

\footnotetext{
a Corresponding author: caoshijie2015@gmail.com
} 


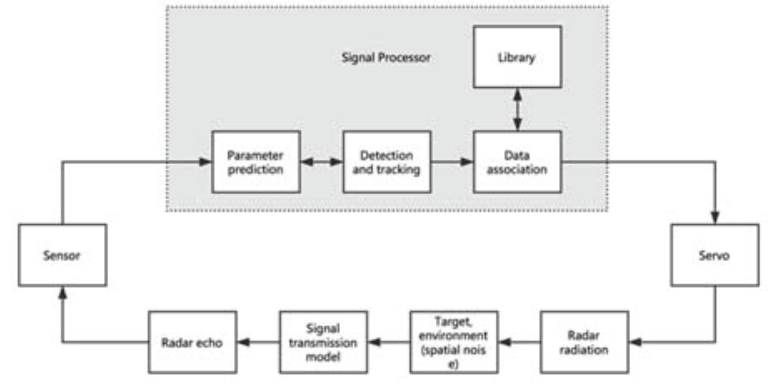

Figure 1. Overview of the processing workflow

At moment $k$, given the state vector $X_{k}$, the observation vector $Y_{k}$ and the value of target state $Y_{0}, \ldots Y_{k}$, it is desirable to estimate the value of the hidden state $X_{k}$. The state vector of the target is $X_{k}=\left[x_{k}, \dot{x}_{k}, y_{k}, \dot{y}_{k}, I_{k}\right]^{T} . \dot{x}_{k}$ shows the rate of change of the variable $x_{k}, I_{k}$ indicates the signal amplitude at moment $k$. If $\dot{x}_{k}=0$, the model is a uniform motion model. Otherwise if $\dot{x}_{k} \neq 0$, it is a motion model with acceleration. The state vector changes according to the state update model, which is described by the following equation:

$$
X_{k}=F\left(X_{k-1}, W_{k}\right)
$$

Where $F$ is the state transition matrix, which is a set of specific stochastic processes that describe the system state noise. The state-transition matrix is

$$
F=\left[\begin{array}{ccc}
F_{S} & 0 & 0 \\
0 & F_{S} & 0 \\
0 & 0 & 1
\end{array}\right]
$$

where

$$
F_{S}=\left[\begin{array}{ll}
1 & T \\
0 & 1
\end{array}\right]
$$

and $T$ is the sampling period.

Given the state vector, the observation vector conforms to a certain posterior distribution and the state vector and the observation vector can be written as follows:

$$
\begin{gathered}
X_{k}=g\left(X_{k-1}\right)+W_{k} \\
Y_{k}=h\left(X_{k}\right)+V_{k}
\end{gathered}
$$

Where $g$ and $h$ are known functions, $W_{k}$ and $V_{k}$ are additive independent additive noise sequences with known probability density function.

\section{Algorithms}

In this paper, an improved sequential smoothing particle filtering(ISS-PF) algorithm is proposed, which aims to reduce the computational consumption. Firstly, the number of particles in the traditional particle filter is reduced, and a small number of sampling points are derived from the proposed distribution of the target. Then, by introducing different weights for each sampling point, after the transformation of the sampling point for smoothing, realtime correction of the filter error ensures that the final output can achieve the target location estimation.

\subsection{Sampling Points}

First, calculate the mean and covariance of the proposed distribution and use them as the basis for the distribution of the sampling points.

$$
\begin{aligned}
\mu_{X} & =\sum x P(x) \\
\operatorname{COV}(X, Y) & =E\left[\left(X-\mu_{X}\right)\left(Y-\mu_{Y}\right)\right]
\end{aligned}
$$

Where $\mu$ is the mean of the proposed distribution. $\operatorname{COV}(X, Y)$ is the covariance of the two variables.

The mean of the proposed distribution is chosen as the first sampling point, and two sets of sampling points are symmetrically selected on both sides of the first sampling point. Furthermore, the distribution of the sampling points conforms to the following constraint equations:

$$
\begin{gathered}
1=\sum_{i} w_{i}^{m} \\
\mu=\sum_{i} w_{i}^{m} f\left(\xi_{i}\right) \\
\sum=\sum_{i} w_{i}^{c}\left(f\left(\xi_{i}\right)-\mu\right)\left(f\left(\xi_{i}\right)-\mu\right)^{\mathrm{T}} \\
\operatorname{COV}(x, y)=\frac{\sum(x-\bar{x})(y-\bar{y})}{n}
\end{gathered}
$$

Where $w_{i}^{m}$ and $w_{i}^{c}$ are the weights of the mean and variance of the first two equations. $f\left(\xi_{i}\right)$ stands for an arbitrary non-linear function and $\xi_{i}$ stands for the $i$ th sampling point.

\subsection{Weights of the Sampling Points}

For convenience, define

$$
\lambda=\alpha^{2}(n+\kappa)-n
$$

The means and variances of all the sampling points in step one are given different weights so that the weights of all the sampling points add up to one, and the formula should satisfy:

$$
\xi_{i}=\left\{\begin{array}{c}
\mu+[\sqrt{(n+\lambda) \Sigma}]_{i}, i=1,2, \ldots, n \\
\mu-[\sqrt{(n+\lambda) \Sigma}]_{i-n}, i=n+1, n+2, \ldots, 2 n
\end{array}\right.
$$

Where the subscript $i$ indicates that the $i$ th column vector of the matrix is selected. This process is equivalent to scaling the covariance matrix with a constant, taking the square root of the matrix and ensuring the symmetry of the sampling points and their weights by adding and subtracting the square root from the mean.

The weight of the mean of the point $\xi_{0}$ is:

$$
W_{0}^{m}=\frac{\lambda}{n+\lambda}
$$

The weight of the variance of the point $\xi_{0}$ is:

$$
W_{0}^{c}=\frac{\lambda}{n+\lambda}+1-\alpha^{2}+\beta
$$


The weights of the mean of the remaining points are the same as the covariance, as described in the following equation:

$$
W_{i}^{m}=W_{i}^{c}=\frac{1}{2(n+\lambda)} \quad i=1,2, \ldots, 2 n
$$

\subsection{Sequential Smoothing}

The sample points are transformed by non-linear function, and the transformed points are smoothed sequentially. First, select a state point as the center so that the other points fall within the area of a circle of radius $r$ and make sure that the distribution of these sampling points consistent with the distribution of the posterior probability of the particle filter:

$$
\hat{p}\left(d x_{k} \mid y_{0: k-1}\right)=\frac{1}{N} \sum_{i=1}^{N} \delta_{\xi_{n}^{i}} d x_{n}
$$

According to the following formula on the sampling points before and after the time to estimate the location of the weighted average

$$
\begin{gathered}
K\left(x_{0}, x_{i}\right)=\exp \left(-\frac{\left(x_{0}-x_{i}\right)^{2}}{2 b^{2}}\right) \\
\hat{f}\left(x_{0}\right)=\frac{\sum_{i=1}^{N} K_{r_{i}}\left(x_{0}, x_{i}\right) f\left(x_{i}\right)}{\Sigma_{i=1}^{N} K_{r_{i}}\left(x_{0}, x_{i}\right)} \\
r_{i}=r_{0} \frac{\left(f\left(x_{i}\right)-\mu\right)^{2}}{\Sigma}
\end{gathered}
$$

Where $K\left(x_{0}, x_{i}\right)$ represents the weight value of the smoothing operation, $\hat{f}\left(x_{0}\right)$ represents the current estimated point of the continuous probability density function of $x, r$ means the smooth window radius and only the point within the interval will participate in the smoothing calculation.

\subsection{Resampling}

After the smoothing procedure, the proposed algorithm then calculates the mean and variance of the smoothed sampling points, re-estimates the proposed distribution based on the mean and variance after smoothing, and resamples from the smoothed distribution function.

The above four steps are repeated until the amount for sequential filtering is sufficient, then the filtering result is outputted.

\section{Simulation Results}

The maneuvering targets move in a square area of $1000 \mathrm{~m} \times 1000 \mathrm{~m}$ with non-linear motions. The sequential time is 30 frames. Set the maximum speed variation to $2 \mathrm{~m} / \mathrm{s}^{2}$. The maximum speed for a target is $100 \mathrm{~m} / \mathrm{s}$. The initial position for the target is $[4.2 \mathrm{~m}, 124.5 \mathrm{~m}]$ and the initial speed for the target is $[1.2 \mathrm{~m} / \mathrm{s}, 1.5 \mathrm{~m} / \mathrm{s}]$. The Monte Carlo simulation is set to 100 times. The power of the noise is $\sigma^{2}=1 / 2$. The defined signal-to-noise ratio is the logarithm of the square-sum of the mean of the signal amplitude to noise power, varying from $0 \mathrm{~dB}$ to $20 \mathrm{~dB}$.

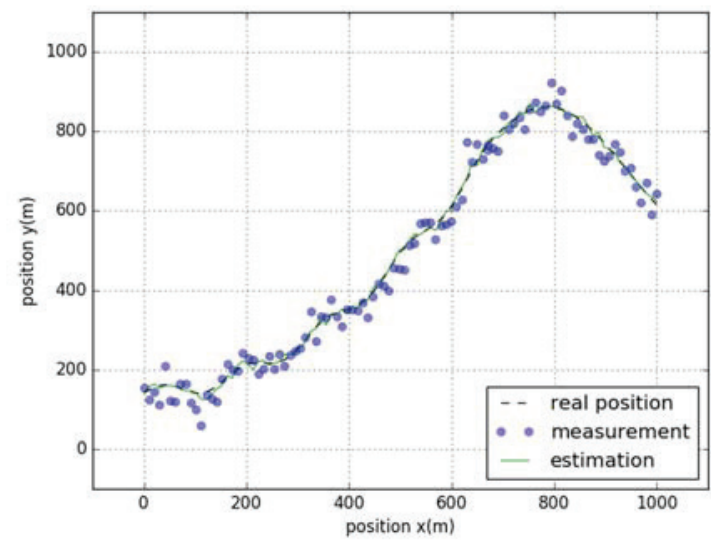

Figure 2. Trajectory track with ISS-PF

Figure 1 shows the resulting trajectory, which is obtained by filtering a group of noisy measurements with ISS-PF. Figure 1 also compares the tracking trajectory and the real track of the weak maneuvering target. Although there are unavoidable errors in a controllable range, especially in the target maneuvering position, the tracking accuracy is high enough to avoid large shifts of the estimation result.

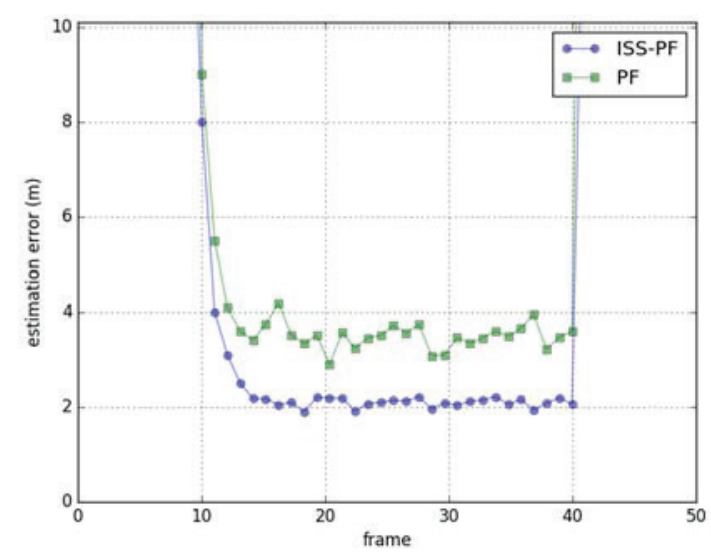

Figure 3. Tracking estimation error

As shown in figure 2, the estimation error is derived by calculating the mean square error along the tracking process. The result shows that ISS-PF algorithm can generally achieve less estimation error of the track than the traditional particle filter algorithm. 


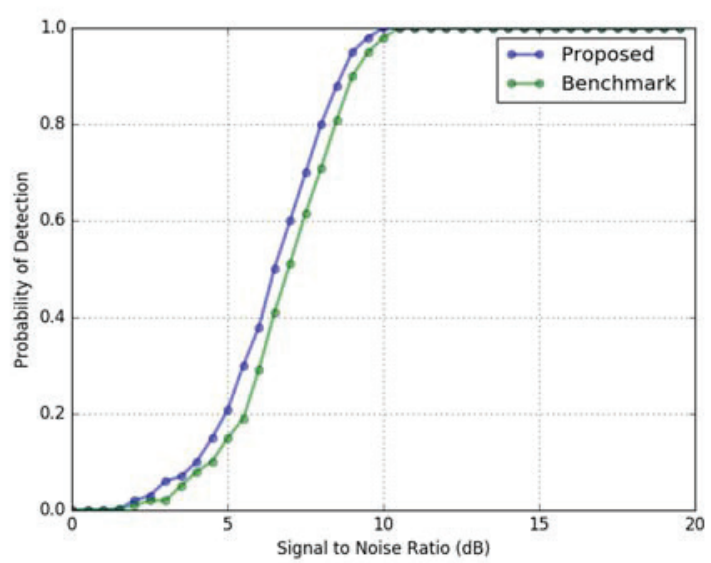

Figure 4. Detection probability comparison

We notice that with the gaining of signal-to-noise ratio, the rise of the detection probability of traditional particle filter is generally slower. On the other hand, the optimized ISS-PF target tracking algorithm can achieve enough detection probability at $6 \mathrm{~dB}$. When the signal-to-noise ratio is above $9 \mathrm{~dB}$, the detection probability can reach more than $90 \%$, and the system can work robustly. Especially when the signal-to-noise ratio is less than $5 \mathrm{~dB}$, the detection probability of the optimized ISS-PF target tracking algorithm is obviously higher than that of the traditional particle filter algorithm.

Table 1. Detection time comparison of the algorithms

Detection time (seconds every 10 simulations)

\begin{tabular}{|c|c|c|}
\hline $\begin{array}{c}\text { Detection } \\
\text { Probability }\end{array}$ & $\begin{array}{c}\text { Traditional } \\
\text { Particle Filter }\end{array}$ & $\begin{array}{c}\text { Proposed ISS- } \\
\text { PF Algorithm }\end{array}$ \\
\hline 0.2 & 402.7803 & 41.4901 \\
\hline 0.4 & 272.1028 & 21.2624 \\
\hline 0.6 & 141.4242 & 10.5848 \\
\hline 0.8 & 30.7465 & 3.5967 \\
\hline 1.0 & 10.0683 & 1.3499 \\
\hline
\end{tabular}

It can be found that the ISS-PF algorithm proposed in this paper is much less costly than the traditional particle filter method in the same simulation environment after reasonable optimization and improvements.

\section{Conclusion}

Summing up, the algorithm of optimization and improvement in this paper can realize the effective tracking and detection of weak maneuvering targets and reduce the overall cost of the system. It can be seen that the advantage of the proposed algorithm in this paper lies in the availability of using less particles to obtain effective estimates. In the case of insufficient system computing resources, high real-time requirements and low accuracy requirements, the ISS-PF algorithm proposed in this paper has some advantages. And in the same condition, the algorithm, compared with traditional target detection and tracking algorithms, shows a superior detection and state estimation performance, especially concerning the detection time.

\section{References}

1. Risfic B, Arulampalam S, Gordon N. Beyond the kalman filter - Book Review[J]. Aerospace \& Electronic Systems Magazine IEEE, 2004, 19(7):3738.

2. Davey S J, Rutten M G, Cheung B. A comparison of detection performance for several Track-BeforeDetect algorithms[C]. International Conference on Information Fusion. IEEE, 2008:1-8.

3. Bandiera F, Besson O, Orlando D, et al. GLRT-Based Direction Detectors in Homogeneous Noise and Subspace Interference[J]. Signal Processing IEEE Transactions on, 2007, 55(6):2386-2394.

4. Vo B N, Vo B T, Mahler R P S. Closed-Form Solutions to Forward-Backward Smoothing[J]. IEEE Transactions on Signal Processing, 2012, 60(1):2-17.

5. Vo B T, Clark D, Vo B N, et al. Bernoulli ForwardBackward Smoothing for Joint Target Detection and Tracking[J]. IEEE Transactions on Signal Processing, 2011, 59(9):4473-4477.

6. Moral P D, Jacod J, Protter P. The Monte-Carlo method for filtering with discrete-time observations[J]. Probability Theory \& Related Fields, 2001, 120(3):346-368.

7. Moral P D, Doucet A, Jasra A. On Adaptive Resampling Procedures for Sequential Monte Carlo Methods[J]. Bernoulli, 2012, 18(1):252-278.

8. Davey S J, Rutten M G, Cheung B. A Comparison of Detection Performance for Several Track-beforeDetect Algorithms[C] International Conference on Information Fusion. IEEE, 2007:1-8.

9. Orlando D, Ricci G, Bar-Shalom Y. Track-BeforeDetect Algorithms for Targets with Kinematic Constraints[J]. IEEE Transactions on Aerospace \& Electronic Systems, 2011, 47(3):1837-1849. 